\title{
Perioperative advantages of laser treatment
}

$\mathrm{N}$ ew data confirm that laser procedures are equivalent to transurethral resection of the prostate (TURP) for symptomatic improvement of benign prostatic hyperplasia (BPH), but reveal superior results when it comes to perioperative outcomes and related morbidity. Compared to TURP, Eraser laser enucleation of the prostate (ELEP) is associated with improved hemostasis, whereas GreenLight HPS 120 W vaporization provides greater preservation of ejaculation. Both methods result in shorter hospital stays and reduced catheterization time.

Lukas Lusuardi and his team at Paracelsus Medical University in Salzburg, report the preliminary results of their experience with ELEP in the Journal of Urology. Successful utilization of the Eraser laser for resection of lung metastases encouraged researchers to test its efficacy in prostate tissue. "Its cutting properties, with convincing hemostatic results, immediately made it our preferred instrument," says Lusuardi.

The 1,318 $\mathrm{nm}$ diode laser has previously been shown to reduce bleeding rates and cause deeper necrosis than other commonly used laser systems, which is thought to be due to the creation of three distinct zones of necrosis-a central crater of vaporized tissue, a broad and light zone of coagulation and a thin corona of hyperemia.

Lusuardi and colleagues randomized 60 men with symptomatic bladder outflow obstruction to undergo either ELEP $(n=30)$ or bipolar TURP $(n=30)$ performed by a single surgeon. Functional outcomes were similar-no significant differences were reported in $\mathrm{Q}_{\max }$, International Prostate Symptom Scores (IPSS) or quality of life between groups, at 1 or 6 months.

The investigators were most interested in evaluating blood loss during surgery. "We think that this aspect is of utmost importance due to the increasing necessity for anticoagulant drugs in the elderly population," explains Lusuardi. Perioperative bleeding was found to be significantly less in men who underwent ELEP; reductions compared to TURP were noted in blood loss $(116.83 \mathrm{ml}$ versus $409.83 \mathrm{ml} ; P<0.05)$, hemoglobin loss $(0.24 \mathrm{~g} / \mathrm{dl}$ versus $0.61 \mathrm{~g} / \mathrm{dl} ; P<0.05)$, and bleeding velocity $(2.90 \mathrm{ml} / \mathrm{min}$ versus $5.49 \mathrm{ml} / \mathrm{min} ; P<0.05)$. In addition,

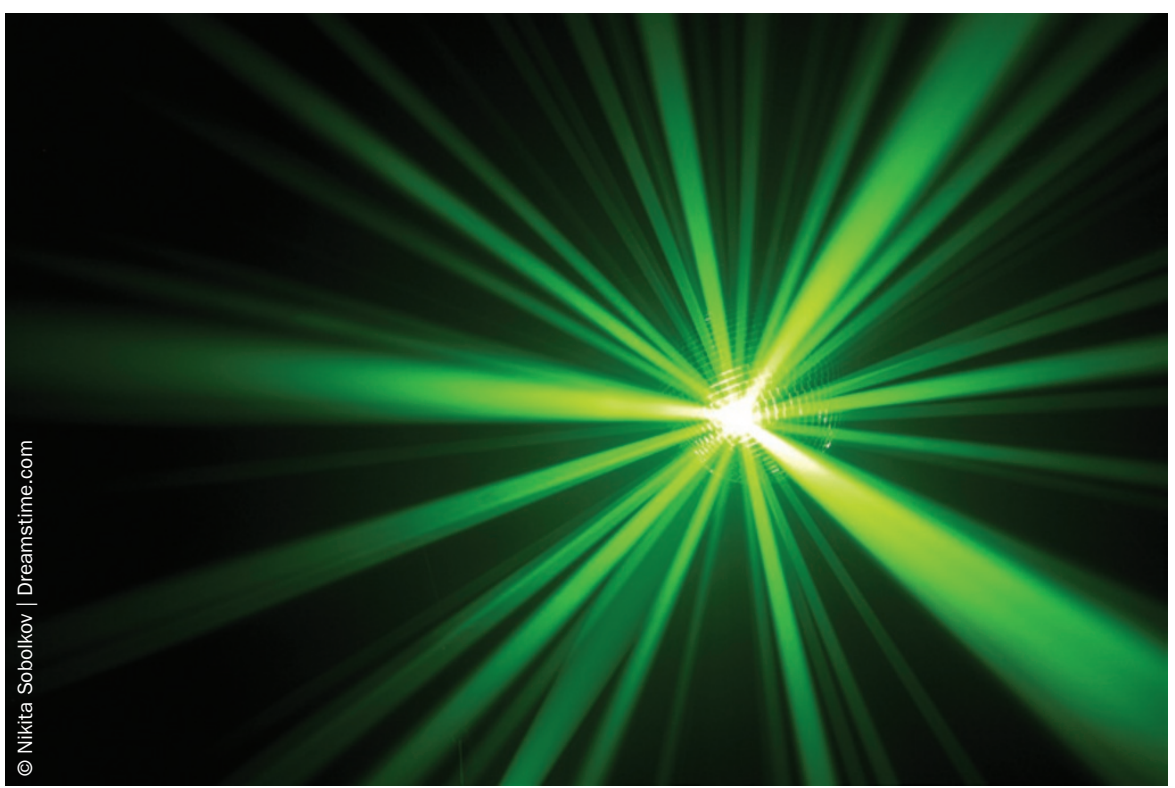

ELEP was performed significantly quicker than TURP (46.7 min versus $60.43 \mathrm{~min} ; P<0.05$ ), and was associated with shorter durations of both indwelling catheterization $(32.80 \mathrm{~h}$ versus $65.73 \mathrm{~h}$; $P<0.05)$ and hospital stay $(45.13 \mathrm{~h}$ versus 91.2 h; $P<0.05)$.

The impressive hemostatic properties of ELEP certainly warrant further study. "We plan to compare ELEP with other lasers in a multicenter setting, as well as investigate reproducibility and the learning curve of this technique," says Lusuardi.

A similar randomized trial was performed by Carlos Capitán and colleagues at King Juan Carlos University in Madrid. They report the outcomes of photoselective vaporization of the prostate (PVP) with a $120 \mathrm{~W}$ GreenLight HPS laser in the October issue of European Urology.

At 2 years after surgery, changes in IPPS, $Q_{\max }$ and quality of life were similar in patients who underwent PVP $(n=50)$ or TURP $(n=50)$. Complication rates were also comparable between groups. However, PVP significantly outperformed TURP in all perioperative parameters measured, including size of catheter, duration of catheterization and hospital stay. Most notably, retrograde ejaculation was reported at a much higher rate in men who underwent TURP, than those who received laser PVP (65\% versus 35\%; $P=0.0001$ ).

Despite these perioperative advantages, until laser enucleation or vaporization techniques are able to provide better symptomatic improvement and volume reduction than resection, bipolar TURP will remain the gold standard treatment option for BPH.

\section{Sarah Payton}

Original articles Lusuardi, L. et al. Safety and efficacy of Eraser laser enucleation of the prostate: preliminary report J. Urol. doi:10.1016/j.juro.2011.07.026 | Capitán, C. et al. GreenLight HPS 120-W laser vaporization versus transurethral resection of the prostate for the treatment of lower urinary tract symptoms due to benign prostatic hyperplasia: a randomized clinical trial with 2-year follow-up. Eur. Urol. 60, 734-739 (2011) 